Journal of

Forensic Research and Crime Studies

\title{
Fast Detection of Recent Cannabis Sativa L. Consumption in Exhaled Breath Us- ing a Mobile Ion Mobility Spectrometer
}

\section{Wolfgang Vautz ${ }^{1,2, *}$ Chandrasekhara Hariharan', Oliver Kayser ${ }^{1,3}$}

${ }^{1} \mathrm{ION}-\mathrm{GAS}$ GmbH, Konrad-Adenauer-Allee 4, 44225 Dortmund, Germany

${ }^{2}$ Leibniz-Institut für Analytische Wissenschaften - ISAS - e.V., Bunsen-Kirchhoff-Straße 11, 44139 Dortmund, Germany

${ }^{3}$ Technische Universität Dortmund, Technische Biochemie, Emil-Figge-Straße 66, 44227 Dortmund, Germany

${ }^{\star}$ Corresponding author: Wolfgang Vautz, ION-GAS GmbH, Konrad-Adenauer-Allee 11, 44263 Dortmund Germany; E-mail: w.vautz@ion-gas.de; Tel: +49-1575-8767712

Received Date: September 12, 2019 Accepted Date: October 22, 2019 Published Date: October 24, 2019

Citation: Wolfgang Vautz (2019) Fast Detection of Recent Cannabis Sativa L. Consumption in Exhaled Breath Using a Mobile Ion Mobility Spectrometer. J Forensic Res Crime Stud 3: 1-11.

\begin{abstract}
The volatile metabolite pattern in exhaled breath after smoking Cannabis sativa L. was determined by ion mobility spectrometry coupled to gas-chromatographic pre-separation (GC-IMS). The aim was to identify typical biomarkers indicating a recent consumption of herbal Cannabis sativa L. In parallel, blood samples were taken and THC, 11-Hydroxy- and 11-Carboxy-THC were analyzed by GC-MS. A high number of volatile constituents - most probably metabolized from the essential oil but not identified as cannabinoids - were detected in the breath. A significant correlation between blood and exhaled breath concentration of THC could not be observed. However, Cannabis sativa L. smoke contains distinct essential oil constituents which may act as indicative biomarkers providing proof for smoking herbal Cannabis sativa L. A characteristic pattern of compounds could be observed using GC-IMS over a detection period of up to $4 \mathrm{~h}$ after last consumption. Method development was carried out successfully leading to a rapid total analysis time of 90 seconds combined with the high userfriendliness of the mobile equipment. In this manuscript, we present the scientific background, the technical implementation and the real-world operation of the commercial prototype.
\end{abstract}

Keywords: Forensic Science; Ion Mobility Spectrometry; Cannabis sativa; Drug Enforcement; Rapid Breath Screening Test

(C)2019 The Authors. Published by the JScholar under the terms of the Creative Commons Attribution License http://creativecommons.org/licenses/ by/3.0/, which permits unrestricted use, provided the original author and source are credited. 


\section{Introduction}

Cannabis (Cannabis sativa $\mathrm{L}$ ) is a psychotropic plant that is frequently used by smoking or oral ingestion for relaxation. Cannabis sativa is the most reported illicit drug identified in impaired drivers in Europe and the US [1]. Driving under the influence of Cannabis causes severe accidents. Nearly $60 \%$ of injured drivers with trauma are tested positive for drugs or alcohol in the US. According to the annual report of the US National Driving Board [2], 11.4\% of Americans and 10\% of Canadians [3] drove in 2010 and 2012 under the influence of illicit drugs. Herbal Cannabis is the most consumed illicit drug worldwide [4] and a strong increase in the number of future drug users can be expected with the on-going legalization. Thus, driving under the influence of Cannabis is an urgent and growing health and traffic security concern. Epidemiologic data $[2,3]$ show that the risk of involvement in a motor vehicle accident is twofold higher after consuming the drug. The primary reason being the dosedependent effect and impairment of cognitive and psychomotoric functions $[5,6]$. As a consequence, drivers under the influence of Cannabis show a higher risk of collision and severe accidents combined with impaired driving skills.

Currently, no valid, rapid, and mobile screening methods are available which fulfil the needs as we know for alcohol checks. Police worldwide use detection devices like wipe tests for sweat or urine tests, based on thin-layer chromatography, which is easy to handle but show low sensitivity, hygienic discomfort and significant error rates in practical use [7-9]. Since cannabis is legalized in many countries for medicinal and recreational use, police are more confronted with drivers of all ages consuming cannabis on a regular basis. Driving under the influence of any drug is still illegal worldwide and countries are starting the debate about thresholds in the range of $1-7 \mathrm{ng} / \mathrm{mL}$ of THC in blood [10]. Rapid test devices will not meet this limit precisely due to the nature of the chemical reaction as a principle proof. Consequently, there is a pressing need for a rapid field test that will enable law enforcement agents, customs officers, and other responsible personnel to obtain presumptive evidence on suspected sample identity.

Today, in 29 countries cannabis is legal for medicinal use and moreover, in Spain, Uruguay and some US states recreational use is legal as well. Similar to other medicinal plants, Cannabis is a phytochemical multi-component mixture with more than 120 cannabinoids including, tetrahydrocannabinolic acid, cannabidiolic acid, cannabigerol acid and cannabichromene acid being known as the most dominant constituents [11]. Over 500 metabolites have already been identified in this species, includ- ing terpenes, sugars, hydrocarbons, steroids, flavonoids, nitrogenous compounds, non-cannabinoid phenols and amino acids $[11,12]$. Moreover, it is clear that besides the dominant THC other constituents have been found to be medicinally active [13]

Cannabis Sativa L. is a typical aromatic plant with a significant concentration of essential oil (approx. 0.2-0.3\%). These, mostly terpenophilic constituents and related human metabolites after application, are volatile and exhaled via the natural breath. Many of the constituents can be detected in the exhaled air which is a boon and a burden at the same time because essential oils consist of 300 up to 1000 compounds [14]. This is why analytics and distinct compound detection and elucidation is complicated. In Cannabis sativa L., about 90 primary constituents in the essential oil are known [15]. But, how to overcome the irresolvable question of compound identification or the best strategy for pattern recognition in the exhaled breath air is one of the key questions in this study.

Various methods for the determination of cannabinoids in plant material have been developed and are summarised by Klein [16]. In the past decades, thin layer chromatography (TLC) was used for cannabinoids separation, combined with colorimetric tests or UV detection under $254 \mathrm{~nm}$ for their identification $[17,18]$. Nowadays, commonly applied techniques are HPLC and GC, and very often coupled with tandem with the mass spectrometric detector. But so far, no one of these sophisticated techniques is mobile and can be used in the field, which would be an outstanding technical advantage.

Ion mobility spectrometry [19] is a powerful technique that fasts, sensitive and mobile and therefore, we investigated its potential concerning detection of drug consumption. The method is based on ion generation and separation of ions in the gaseous phase under an electric field [20,21]. This technique separates ions in an electric field by size and shape due to collisions with a drift gas on their way to a detector. IMS is a very powerful and extremely sensitive technique to detect volatile molecules at $\mathrm{ppt}_{\mathrm{v}}$ concentration ranges. In combination with gas-chromatographic pre-separation (GC-), extremely complex mixtures such as exhaled breath can be comprehensively analyzed quantitatively. IMS and GC-IMS instruments are currently used in various technical fields like explosive detection at airport security checks [22], chemical warfare agents [23], food control [24], process control [25] and biomedical analysis [26-28], illicit drugs [29], and forensics [30].

This study aimed to investigate if cannabinoids and other characteristic volatile compounds of the psychotropic plant can be identified in exhaled breath by GC-IMS and to find a cor- 
relation between the blood concentrations and detected volatile THC or other relevant biomarkers. Therefore, additional blood sampling was conducted and analyzed for the presence of THC, 11-Hydroxy- and 11-Carboxy-THC.

\section{Materials and Methods}

\section{Plant Material}

Cannabis sativa L. variety Bedrocan was supplied by Bedrocan BV (The Netherlands). The plants were grown indoors under standardized conditions as explained in a previous report [31]. The saplings were initially generated from cuttings of standardized mother plants and cultivated under controlled, long daylight conditions (18 h/day). After the vegetative growth phase, the flowering stage was induced under a shorter ( $12 \mathrm{~h} /$ day) light regime for eight weeks. The trichomes were isolated and analyzed for THC content from week 5 to week 8 of the flowering period. The plant specimen was assigned the voucher number Bedrocan: N5.26.06.2012. All plant handling and experimental procedures were carried out under license No. 4584989, issued by the Federal Institute for Drugs and Medical Devices (BfArM), Germany.

\section{Chemicals}

All chemicals were obtained from Sigma-Aldrich in the highest available purity.

\section{GC-IMS - gas-chromatography coupled to ion mobility spectrometry}

A custom-made ion mobility spectrometer coupled to a multi-capillary gas-chromatographic column (GC-IMS, ISAS, Dortmund, Germany) was applied for the present investigations [26]. Ion mobility spectrometry is based on the characteristic mobility of ions in an electric field under a counter-current of a neutral drift gas [19]. The ions collide with the drift gas molecules, thus being separated based on size and shape.

Measuring the drift time of the ions enables the calculation of the drift velocity. The normalization of the velocity to electric field strength and to temperature and pressure results in the so-called reduced ion-mobility [19] (Eq. 1). This value is characteristic of a particular ion.

$$
K_{0}=\frac{L_{D}}{E \cdot t_{D}} \cdot \frac{p}{p_{0}} \cdot \frac{T_{0}}{T}
$$

Where $\mathrm{K}_{0} \quad$ reduced ion mobility in $\mathrm{cm}^{2} \mathrm{~V}^{-1} \mathrm{~s}^{-1}$
E
electric field strength in $\mathrm{Vcm}^{-1}$
$\mathrm{L}_{\mathrm{D}} \quad$ drift path length in $\mathrm{cm}$
$t_{D} \quad$ drift time in $s$
$\mathrm{p} \quad$ pressure in $\mathrm{hPa}, \mathrm{p}_{0}=1013.2 \mathrm{hPa}$
$\mathrm{T} \quad$ temperature in $\mathrm{K}, \mathrm{T}_{0}=273.2 \mathrm{~K}$

When analyzing complex, humid gas samples such as exhaled breath, clustering of the different analyte ions and water molecules takes place, thus making their identification difficult or even impossible. Therefore, an additional separation step using gas-chromatographic columns are applied before the sample is introduced into the IMS. For the present study, a $20 \mathrm{~cm}$ multicapillary column operated at $40^{\circ} \mathrm{C}$ (Sibertech, Novosibirsk, Russia) was used.

Data acquisition was carried out with qIMS, a custommade software from ISAS, Dortmund, Germany. Data interpretation was carried out with the software tool IONysos (ION-GAS $\mathrm{GmbH}$, Dortmund, Germany). This tool enables data treatment, baseline corrections, smoothing and normalization of the signal intensity. The characteristic peaks in the 3-dimensional GC-IMS dataset (signal intensity vs. ion mobility and retention time) are indicated and can be assigned to specific compounds by comparison with a substance database. The signal intensity can be attributed to a specific concentration after a calibration. Finally, the data from different GC-IMS analyses be compared automatically.

Exhaled breath sampling was carried out by exhalation through a mouthpiece with a flow sensor (differential pressure measurement). If the volunteer exhales, the sample flow is drawn from the main exhalation stream at a rate of $100 \mathrm{ml} / \mathrm{min}$ [32].

\section{GC-MS Analysis}

Qualitative and quantitative analyses of THC, 11-OHTHC and THC-COOH were carried out at Forensische Toxikologie in the InstitutfürRechtsmedizin of the Westfälische-WilhemsUniversität, Münster, Germany with a validated method using gas chromatography-mass spectrometry following solid-phase extraction. $1 \mathrm{~mL}$ plasma was diluted with $2 \mathrm{~mL}$ phosphate buffer (0.1 M, pH 6). After the addition of $50 \mu \mathrm{L}$ I.S. working solution (5 ng THC-d3, 5 ng 11-OH-THC-d3 and 50 ng THC-COOH-d9), $250 \mu \mathrm{L}$ acetonitrile was added under vortex-mix and centrifuged (2576 x g, $10 \mathrm{~min}$ ). The resulting supernatant was decanted to conditioned chromabond Drug II columns (Macherey-Nagel). After sample application, the columns were washed with $2 \times 3$ $\mathrm{mL}$ water, followed by two drying steps of $5 \mathrm{~min}$ in a stream of nitrogen with a pre-wash of $100 \mu \mathrm{L}$ acetone in between. After the 
elution of THC and 11-OH-THC with $1.6 \mathrm{~mL}$ acetone, each column was washed with $2 \mathrm{~mL}$ acetonitrile/0.1 M acetic acid (30:70, $\mathrm{v} / \mathrm{v}$ ) followed by a drying step of $10 \mathrm{~min}$ in a stream of nitrogen. After the addition of $1 \mathrm{~mL}$ hexane and $1 \mathrm{~mL}$ hexane/ethyl acetate $(70: 30, v / v)$ the columns underwent a final drying step of $5 \mathrm{~min}$ in a stream of nitrogen before the final washing step of 2 $\mathrm{mL}$ acetone. THC-COOH was finally eluted with acidic acetone (0.05 $\mathrm{M}$ acetic acid in $1.6 \mathrm{~mL}$ acetone) into the same collecting tube as THC and 11-OH-THC. After evaporation to dryness at $40^{\circ} \mathrm{C}$ in a stream of nitrogen, the extracts were reconstituted with $30 \mu \mathrm{L}$ MSTFA (N-methyl-N-(trimethylsilyl) trifluoroacetamide) for derivatization at $70^{\circ} \mathrm{C}$ for $20 \mathrm{~min}$. The GC-MS analysis was carried out with an Agilent Technologies 7890A gas chromatographic system equipped with a mass selective detector 5975C and an auto-sampler 7693 was additionally used. Detection was accomplished with the mass selective detector operating in electron impact (EI) selective ion monitoring (SIM) mode. The GC separation was carried out with a capillary column OPTIMA 5 MS Accent (95\% dimethylsiloxane, 5\% diphenyl siloxane, $30 \mathrm{~m}$ x $0.25 \mathrm{~mm}$ i.d. $\mathrm{x} 0.25 \mu \mathrm{m}$ ) with the following temperature program: $1.5 \mathrm{~min}$ at $150^{\circ} \mathrm{C}, 9^{\circ} / \mathrm{min}$ up to $260^{\circ} \mathrm{C}$, hold for $6 \mathrm{~min} ; 30^{\circ}$ min up to $300^{\circ} \mathrm{C}$, hold $8 \mathrm{~min}$. The helium gas flow rate was 1.0 $\mathrm{mL} / \mathrm{min}$. The temperatures of the injector, the transfer line, the ion source, and the quadrupole were $250^{\circ} \mathrm{C}, 280^{\circ} \mathrm{C}, 230^{\circ} \mathrm{C}$ and $150^{\circ} \mathrm{C}$ respectively. The injection mode was splitless, and the injection volume $2 \mu \mathrm{L}$. The $\mathrm{m} / \mathrm{z}$ values used for identification and quantification of the trimethylsilyl derivates in the SIM-mode were as follows (target ion underlined): THC-d3 374, 389; THC 371, 386, 303; 11-OH-THC-d3 374, 462; 11-OH-THC 371, 459, 474; THC-COOH-d9 380, 306; THC-COOH 371, 297, 473. Analytical limits of the method (LOD/LOQ) are for THC (0.15 ng/ $\mathrm{mL} / 0.3 \mathrm{ng} / \mathrm{mL}), 11-\mathrm{OH}-\mathrm{THC}(0.20 \mathrm{ng} / \mathrm{mL} / 0.30 \mathrm{ng} / \mathrm{mL})$ and for THC-COOH (2.0ng/mL/3.0 ng/mL).

\section{Experimental Setup}

First, Cannabis sativa Flos including different varieties, (Cannabis sativa L. var., Bedica var., Bedrobinolvar., Bedrocan) was investigated by analyzing the headspace of cannabis by GCIMS as a positive control. Additionally, a reference standard of THC was analyzed as well. Before the start of the inhalation experiment, the natural breath and a blood sample of both volunteers (V1/V2) were recorded as a negative control. Exhaled breath was analyzed by IMS. Volunteers were asked to exhale through the mouthpiece of the GC-IMS and $8 \mathrm{~mL}$ breath was injected into the analytical system. Both volunteers inhaled a single dose of $0.5 \mathrm{~g}$ of dried herbal Cannabis Sativa L. flos and exhaled through the mouthpiece into the GC-IMS. An injection volume of $8 \mathrm{~mL}$ breath. Both inhalation experiments and blood samples were taken over a time period of $240 \mathrm{~min}$ and $406 \mathrm{~min}$, respectively.

\section{Ethics \& legal situation}

All volunteers inhaling herbal Cannabis sativa L. flos were legal users due to medical conditions. They gave written informed consent in the frame of a positive ethics committee vote (IfADo, Dortmund, No. 66-01.07.2013). The data was recorded, stored, and evaluated anonymously.

The ethics committee vote allowed us to analyze breath samples of volunteers without applying any medication or drug which are used by them anyways due to their medical prescriptions. Therefore, in the frame of the present study, only medical Cannabis was consumed by the volunteers. Furthermore, the legal situation in Germany, where the study was carried out, allows only the application of medical Cannabis.

\section{Results and Discussion}

The analysis of blood samples by GC-MS showed that the volunteers were not under the influence of cannabis at the beginning of the test. Maximum THC concentrations were reached shortly after consumption; $8 \mathrm{ng} / \mathrm{mLand} 10 \mathrm{ng} / \mathrm{mL}$ for the two volunteers and were detectable up to 5 hours post consumption. 11-OH-THC was detectable up to 2-3 hours and THC-COOH up to ca. 6 hours (Table 1).

The analysis of the headspace of herbal material found that different brands resulted in slightly different GC-IMS chromatograms (see Figure 1). The differences are not only in the overall intensity but also in the relation of the different signal intensities. However, most of the detected signals could be found in the headspace analysis data of all cannabis samples. In the current study, only medical cannabis was used by the volunteers due to the restrictions of the ethics committee and considering the legal situation in Germany. But in view of the similarities in the peak pattern from different types and brands of Cannabis, similar results from breath analyses can be expected as well. 


\begin{tabular}{|c|c|c|c|}
\hline \multicolumn{5}{|c|}{ Volunteer 1} \\
\hline $\begin{array}{c}\text { time after cannabis consumption } \\
(\mathrm{min})\end{array}$ & THC (ng/mL) & $11-\mathrm{OH}-\mathrm{THC}(\mathrm{ng} / \mathrm{mL})$ & THC-COOH (ng/mL) \\
\hline 0 & n.d. & n.d. & n.d. \\
\hline 26 & 8,0 & 0,33 & 4,3 \\
\hline 36 & 6,1 & 0,37 & 4,7 \\
\hline 46 & 5,1 & 0,34 & 4,1 \\
\hline 56 & 4,4 & 0,36 & 3,6 \\
\hline 66 & 4,7 & 0,42 & 3,6 \\
\hline 106 & 3,4 & 0,40 & 3,2 \\
\hline 136 & 1,9 & 0,32 & 3,3 \\
\hline 316 & 0,30 & n.d. & 2,6 \\
\hline 406 & n.d. & n.d. & n.d. \\
\hline
\end{tabular}

\begin{tabular}{|c|c|c|c|}
\hline \multicolumn{5}{|c|}{ Volunteer 2} \\
\hline $\begin{array}{c}|c| \\
\text { time after cannabis consumption } \\
(\mathrm{min})\end{array}$ & THC (ng/mL) & $11-\mathrm{OH}-\mathrm{THC}(\mathrm{ng} / \mathrm{mL})$ & THC-COOH (ng/mL) \\
\hline 0 & n.d. & n.d. & n.d. \\
\hline 43 & 10 & 1,3 & 9,9 \\
\hline 53 & 5,6 & 1,0 & 10 \\
\hline 63 & 5,4 & 0,98 & 7,7 \\
\hline 73 & 4,9 & 0,93 & 7,4 \\
\hline 83 & 3,6 & 0,87 & 6,3 \\
\hline 123 & 3,0 & 0,71 & 5,8 \\
\hline 153 & 1,9 & 0,58 & 5,0 \\
\hline 303 & 0,39 & n.d. & 3,1 \\
\hline 393 & n.d. & n.d. & 3,6 \\
\hline
\end{tabular}

Table 1 Concentrations in a toxicokinetic blood profile of 2 volunteers recorded by GC-MS.

Cannabis sativa $\mathbf{L}$.

var. Bedrocan (a)
Cannabis sativa $\mathbf{L}$. unknown origin (b)

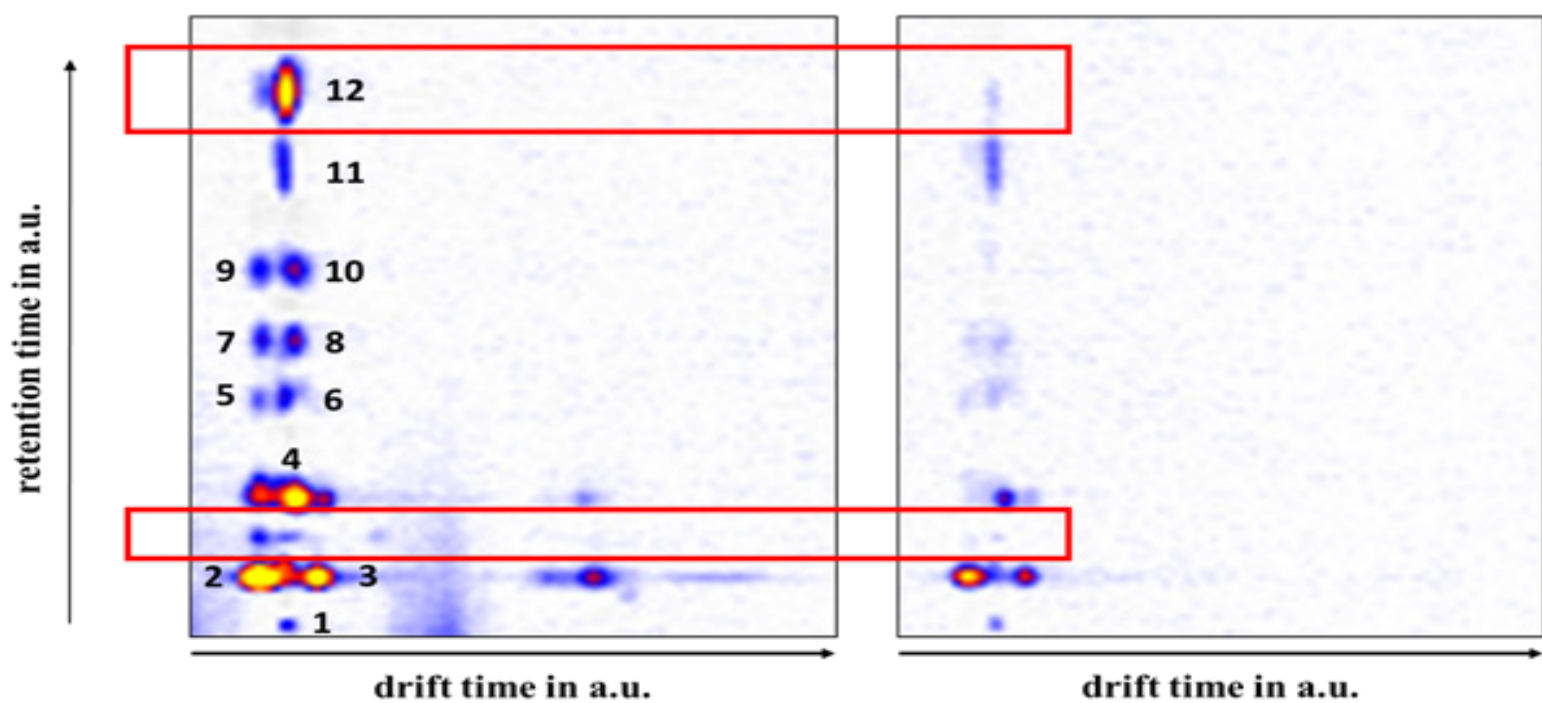

Figure 1 Pattern of Cannabis sativa L. var. Bedrocan (a) and one of unknown origin (b). The 12 signals used for detection of cannabis Consumption in breath are numbered and 2 exemplary differences are indicated by a red frame. 
Before starting with the analysis of exhaled breath from cannabis consumers, the samples itself were investigated. For the purpose, the headspace of cannabis was introduced into the GC-IMS. It was found that different brands resulted in slightly different GCIMS chromatograms (see Figure1). However, most of the detected signals could be found in the headspace analysis data of all cannabis samples.

In comparison with reference compounds, no THC or related human metabolites were detected. However, the GC-IMS data obtained showed clear peaks related to the uptake of essential oil constituents which were present even after 240 min p.a.

\section{Cannabis sativa $\mathrm{L}$.} var. Bedrocan, headspace (a)

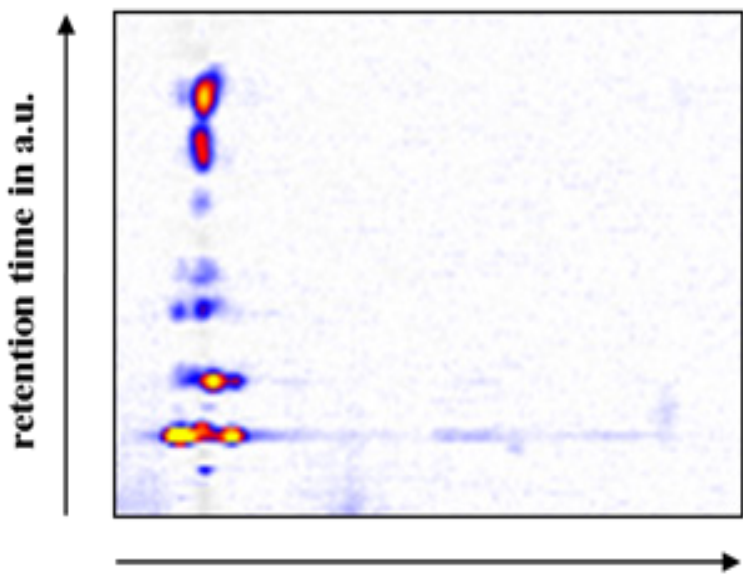

drift time in a.u.
(Figure 2). Before administration of dried herbal Cannabis sativa L. flost he headspace and natural breath of both volunteers were recorded as a positive control for further in vivo methods. Besides the many unknown peaks, a characteristic pattern of 3 peaks - peak 1, 2 and 4 as also detected in the headspace of the cannabis samples (see Figure 1) was indicative for smoking herbal medicinal cannabis and was significant over $240 \mathrm{~min}$.

The slope of one of the relevant signals over 4 hours after smoking is presented in Figure 3. Other peaks from the pattern in Figure 1 was also detected in the first breath analysis, 10 min after administration, but disappeared over time.

\section{Exhaled breath of V1 after smoking (b)}

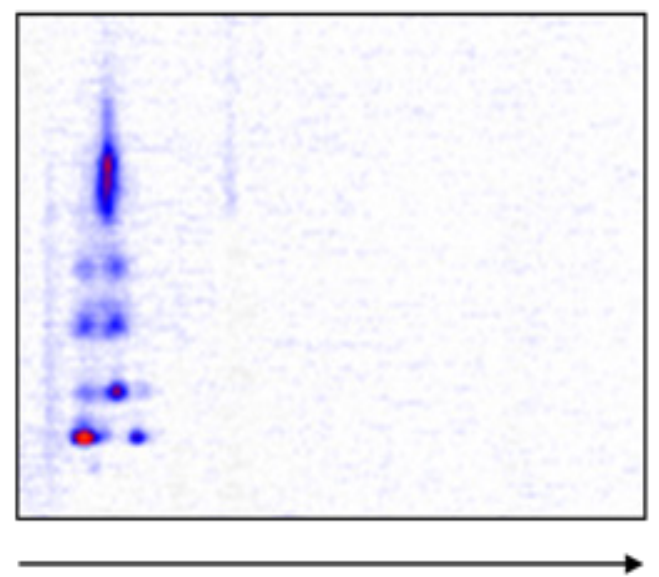

drift time in a.u.

Figure 2 GC-IMS signal pattern of Cannabis sativa L. var. Bedrocan: headspace of a dried sample (a) and after smoking (0.5 g) by $\mathrm{V} 1(\mathrm{~b})$.

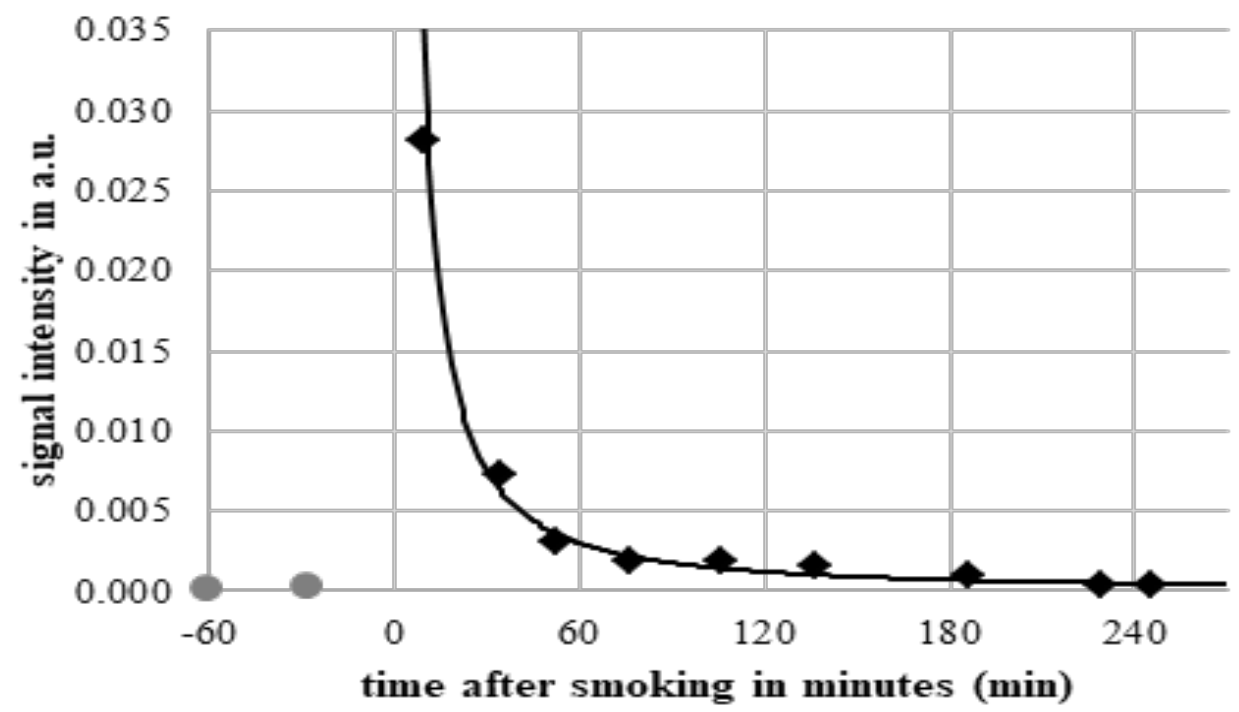

Figure 3 GC-IMS signal intensity of one particular Cannabis sativa L. biomarkers (peak no. 1 as indicated in Fig. 1a) overtime before $(\bullet)$ and after smoking $(\diamond)(0.5 \mathrm{~g})$. 
By algorithm design, 12 peaks were identified in total to be significant for positive proof of cannabis consumption and to discriminate between smoking, eating legal hemp food products (hemp oil, hemp cookies, hemp beer etc.) and to eliminate false positive cannabis detection by consumption of other plants like carrots (Daucus carota). Indeed, none of the before mentioned products produced any of the 12 peaks mentioned in Fig. 1 as a cannabis pattern. After the consumption of $1 \mathrm{~kg}$ of carrots, a significant caryophyllene oxide signal could be detected in exhaled breath. This compound is sometimes mentioned as a compound for training dogs for cannabis search. However, we found this compound in Cannabis headspace only to a very low extent.

IMS can be considered as a highly sensitive analytical technique for the detection of cannabis biomarkers after smoking, even if THC as an illicit compound cannot be detected directly. This is critical for drug enforcement because a direct proof is missing, but using 12 discriminating peaks from the recorded spectrum, a highly positive indication is obtainable. Identification of some of the peaks is ongoing, but the presence of e.g. caryophyllene oxide as one peak is proven and it is important as a biomarker (Figure 4). Due to the presence of caryophyllene oxide in other plants or food products (e.g. carrots or hop) false-positive proofs are, in principle, possible. In a second experiment, $\mathrm{V} 1$ ate $1 \mathrm{~kg}$ fresh carrots and besides caryophyllene oxide (Figure 4) no significant peak pattern as found from cannabis consumption was detected. In our ongoing study, both volunteers ate, over three days, hemp-based food products like pasta, sauces, cannabis oil, sweets, etc, but no cannabinoids were detected by IMS before and after food uptake.
No direct proof of THC was possible, but in headspace studies with a methanolic THC solution $(5 \mathrm{mg} / \mathrm{mL})$ the illicit drug was detected at concentrations of at least $1 \mathrm{ng} / \mathrm{mL}$ and below (data not presented). These data suggest that failure detection is not due to the malfunction of the IMS detector itself. A major reason for not detecting THC is the fact, that this compound is not volatile at the body temperature of $37^{\circ} \mathrm{C}$. Therefore, it can be detected in breath only in the first minutes after smoking, being still present as a product of the cannabis pyrolysis.

Furthermore, fast clearance in the body with a distribution time of 8-10 min and half lifetime for THC of $\mathrm{T}_{50}=30$ min gives an initial explanation as to why detection after 1 hour of smoking is critical and not easy [33]. Most of THC uptake (150 mg/cigarette) is fully absorbed and will not diffuse from the blood into the respiratory air. Currently used on-site rapid drug screening tests like wipe tests or urine tests have a detection limit of $2 \mathrm{ng} / \mathrm{mL}$. It must also be pointed out that there is a high false-positive/false negative error rate of on-site urine, oral fluid or sweat tests (10-15\%). This is due to a failed detection at higher concentrations above $50 \mathrm{ng} / \mathrm{mL}$ [31]. Kintz et al. 2005 stated in their technical note [34] that some drug wipe on-site test is critical at high concentration and they are not safe at low concentrations around 1-2 ng/mL. Another important point is the total evaluation time. The total time required for a first result after sampling with on-site tests, depending on the test, is between 3-12 $\mathrm{min}$ [35]. Here, IMS is extremely competitive with an evaluation time of 1-2 min depending on a single peak or full pattern analysis.

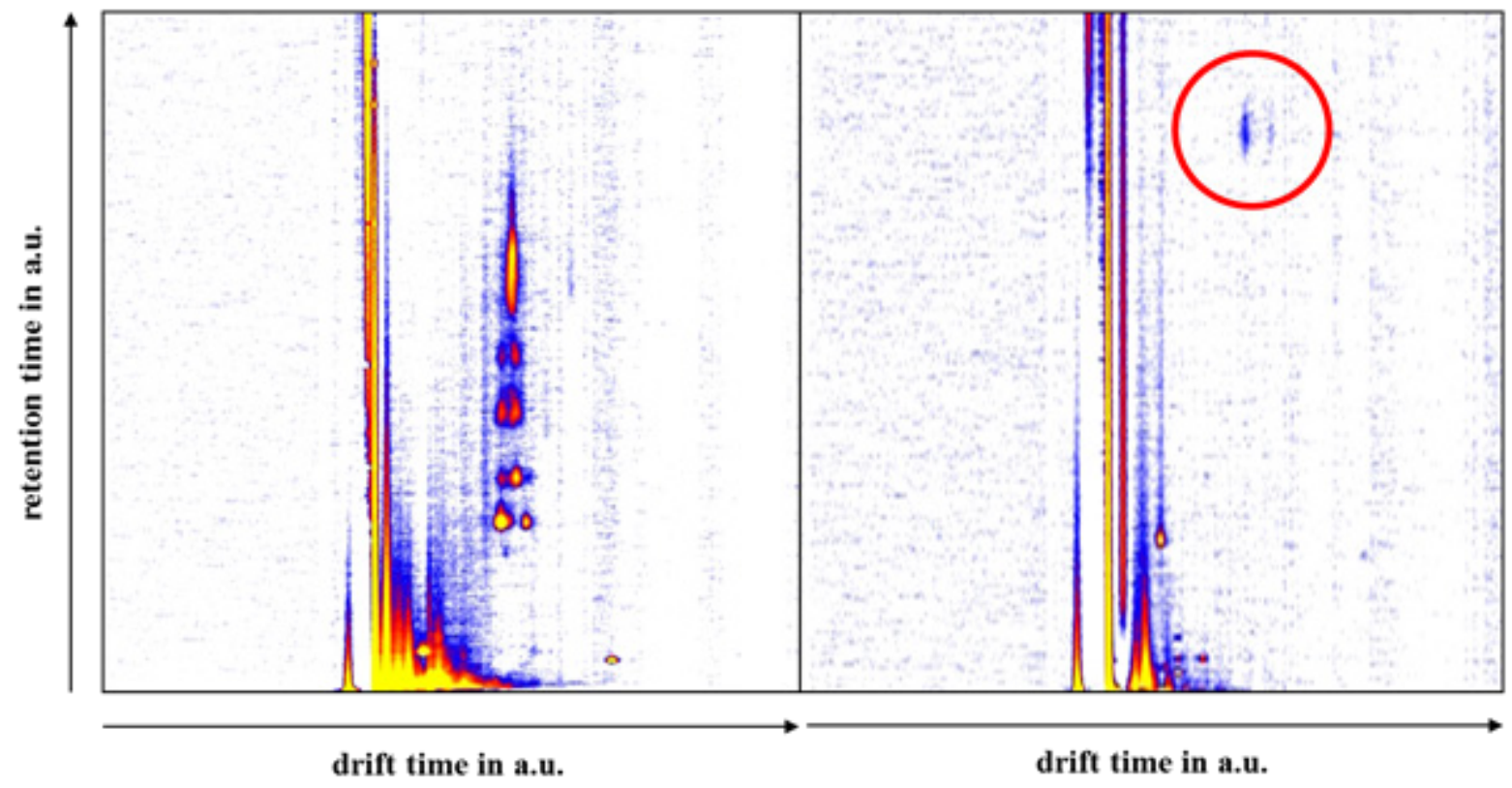

Figure 4 Pattern of Cannabis sativa L. var. Bedrocanafter smoking $0.5 \mathrm{~g}$ (a) and after eating $1 \mathrm{~kg}$ of Daucus carota by V1 (b), the red circle indicates the presence of caryophyllenexpoxid. 


\section{Conclusions \&Real-World Operation}

Ion mobility spectrometry coupled to gas-chromatographic pre-separation (GC-IMS) is a powerful analytical method that allows the detection of Cannabis metabolites in the exhaled air even 240 min after consumption of the drug. Cannabinoids such as THC were not detected by IMS from exhaled breath as they are not volatile at $37^{\circ} \mathrm{C}$ body temperature. The remaining essential oil pattern after smoking cannabis is an indirect proof because of significant exhalation over a time period of $240 \mathrm{~min}$, which can be considered as the critical time for safe driving. At least 12 indicative biomarkers were identified to detect drivers having smoked herbal cannabis in the indicated time frame with a false positive error that is superior to all other rapid and mobile screening tests currently used by the drug enforcement. Due to the mentioned time frame of approx. $4 \mathrm{~h}$, positive detection of those biomarkers suggests, that a detection limit of $1 \mathrm{ng} / \mathrm{mL}$ THC could be met, however, this must be validated still by blood tests. To develop IMS as a mobile and fast screening device, the next step must be the identification of all detected biomarkers and to validate these results in field studies with the police or other drug enforcement organizations.

It has to be kept in mind, that due to the restrictions from the ethics committee vote, only medical cannabis could be applied to the volunteers for the experiments described in this study. However, due to the fact, that the pattern detected in the headspace of medical and "street" cannabis is very similar to differences in the signal intensities of certain peaks only, it can be expected, that this will also result in similar patterns in exhaled breath after consumption. Certainly, further studies with more volunteers and different types of cannabis - e.g. in countries where medical and recreational consumption is legal - have to be carried out for validation of the method presented here. Furthermore, other pathways for consuming Cannabis such as eating cannabis cookies should also be investigated in the future as a significantly different metabolic process will influence the possible detection in exhaled breath.

The instrument used includes an internal circuit for the operational gas and a battery, thus allowing autonomous operation for up to 4 hours (see Figure 5). This mobile GC-IMS named ION drug is currently commercially available. The operation does not require any special training. The user-interface informs about the instruments' status, e.g. for being ready for operation after a short warm-up phase. After the start of an analysis run via the touch screen of the instrument, the user is then guided through the procedure of controlled sampling, analysis after successful sampling and - if sampling was not successful - on a repetition of the procedure (see Figure 6). After the complete analysis, which takes 90 seconds, the operator is then given the information if recent cannabis consumption was determined or not (see Figure 6) using the analogy of a traffic light. However, the comprehensive raw data are stored internally for a possible detailed examination later on.

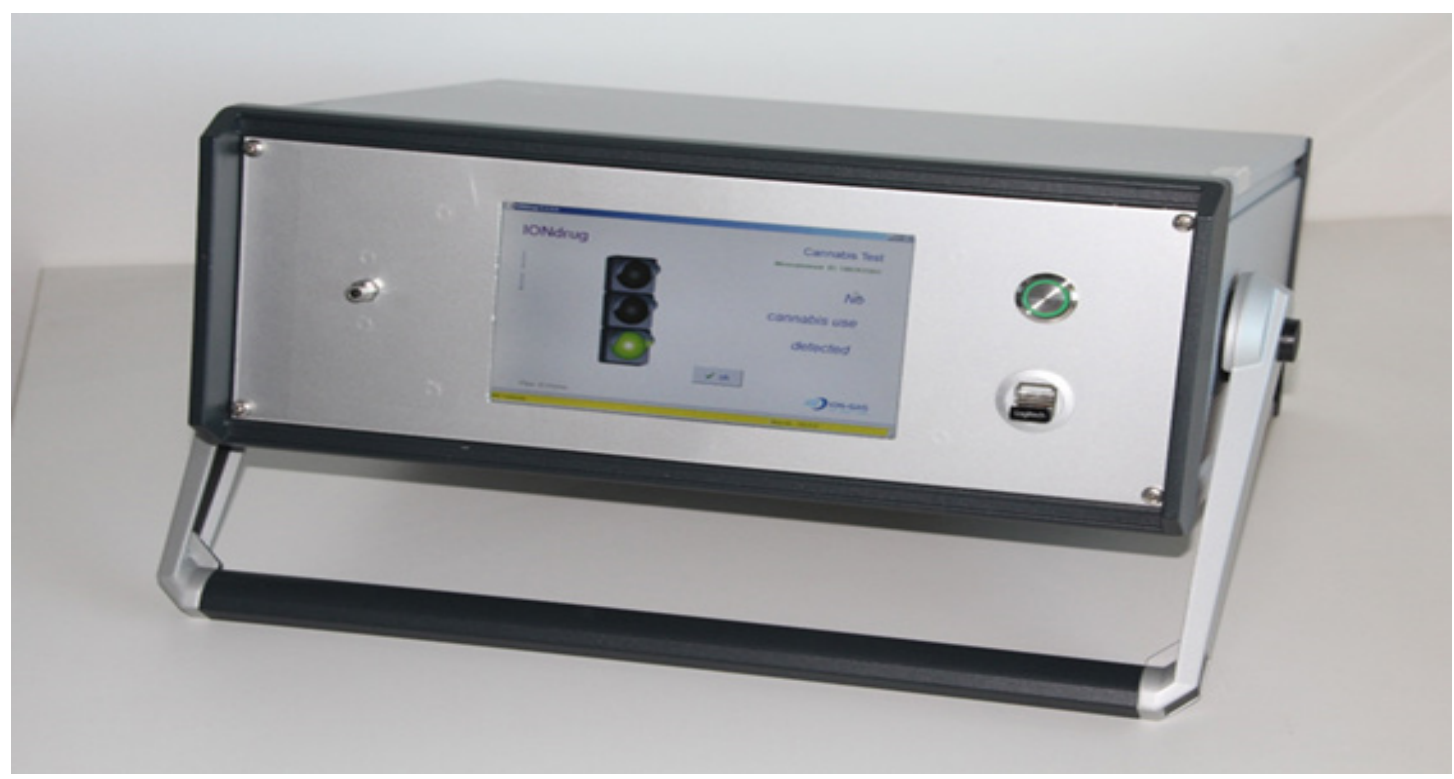

Figure 5 The mobile GC-IMS IONdrug with $\sim 8 \mathrm{~kg}$ incl. a battery for $4 \mathrm{~h}$ autonomous operation. 


\section{Sampling \& Analysis}
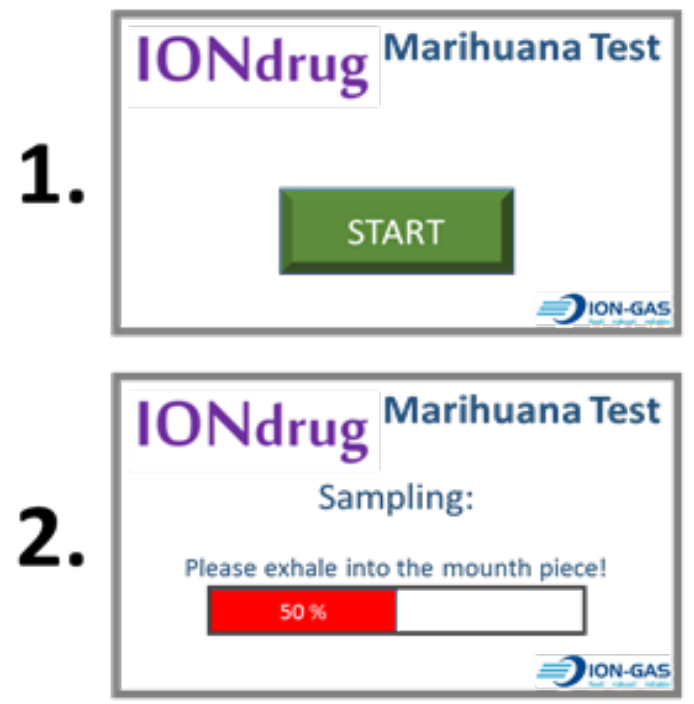

Result Display

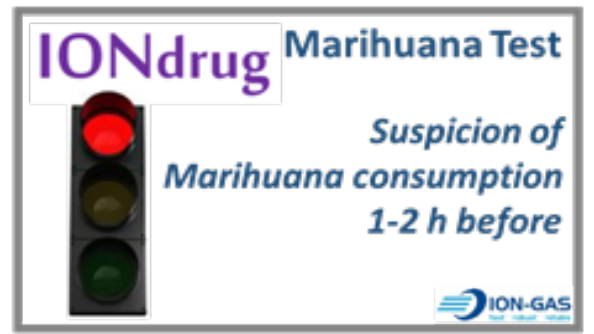

4.

$O R$

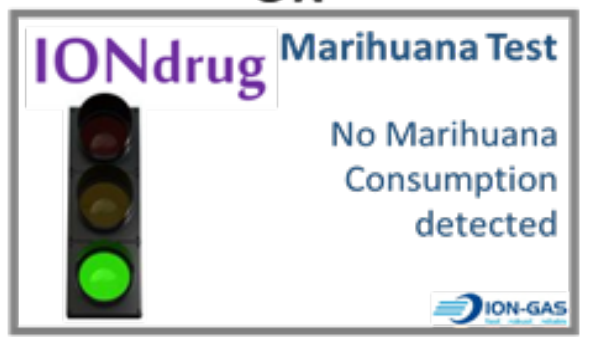

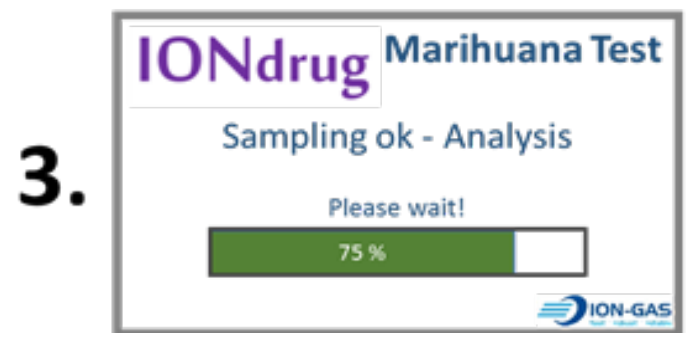

Figure 6 Exemplary screenshots of a complete run of cannabis consumption detection in exhaled breath.

\section{Acknowledgments}

This research was supported by the German Federal Ministry for Economics in the Exist Programme (03EFANW0066). Furthermore, the financial support of the Bundesministerium für Bildung und Forschung and the Ministerium für Innovation, Wissenschaft und Forschung des Landes Nordrhein-Westfalen is gratefully acknowledged. We thank Nouman Al Mofti for supporting us with lab work. Furthermore, analysis carried out by the Westfälische-Wilhems-Universität, Universitätsklinikum Münster, Institut für Rechtsmedizin, Forensische Toxikologie, Münster, Germany is gratefully acknowledged. 


\section{References}

1) Bondallaz, P, Favrat, B, Chtioui, H, Fornari, E, Maeder, P, Giroud, C (2016) Cannabis and its effects on driving skills. Forensic Science International 268: 92-102.

2) Hartman RL, Brown TL, Milavetz G, Spurgin A, Pierce RS, Gorelick DA, et al. (2016) Cannabis effects on driving longitudinal control with and without alcohol. Journal of Applied Toxicology 36: 1418-1429.

3) Wettlaufer A, Florica RO, Asbridge M, Beirness D, Brubacher J, et al. (2017) Estimating the harms and costs of cannabis-attributable collisions in the Canadian provinces. Drug and Alcohol Dependence 173: 185-190.

4) UNDOC - United Nations Office on Drugs and Crime (2017) World Drug Report Retrieved from https://www.unodc. org/wdr2017/field/Booklet_3_Plantbased.pdf\%0Ahttp://www. unodc.org/unodc/secured/wdr/wdr2013/World_Drug_Report_2013.pdf

5) Asbridge M, Hayden JA, Cartwright JL (2012) Acute cannabis consumption and motor vehicle collision risk: systematic review of observational studies and meta-analysis. BMJ 344: e536.

6) Neavyn MJ, Blohm E, Babu KM, Bird SB (2014) Medical marijuana and driving: a review. Journal Medicine and Toxicology 10: 269-279.

7) Musshoff F, Madea B (2006) Review of Biologic Matrices (Urine, Blood, Hair) as Indicators of Recent or Ongoing Cannabis Use. Therapeutic Drug Monitoring 28: 155-163.

8) Verstraete AG (2005) The Results of the Roadside Drug Testing Assessment Project. In: Wong RC, Tse HY (eds.) Drugs of Abuse. Forensic Science and Medicine, Humana Press: 271292.

9) Samyn N, De Boeck G, Verstraete AG (2002) The use of oral fluid and sweat wipes for the detection of drugs of abuse in drivers. J Forensic Sci. 47:1380-7.

10) Grotenhermen F, Leson G, Berghaus G, Drummer OH, Krüger HP, et al. (2007) Developing limits for driving under cannabis. Addiction 102: 1910-1917.

11) ElSohly MA, Radwan MM, Gul W, Chandra S, Galal A (2017) Phytochemistry of Cannabis sativa L. In: Progress in the chemistry of organic natural products. 1-36.

12) ElSohly MA, Slade D (2005) Chemical constituents of marijuana: The complex mixture of natural cannabinoids. In: Life Sciences. 539-548.

13) Tuner S, Williams C, Iversen L, Whalley B (2017) Molecular Pharmacology of Phytocannabinoids. Progress in the Chemistry of Organic Natural Products 103: 81-101.

14) Dhifi W, Bellili S, Jazi S, Bahloul N, Mnif W (2016) Es- sential Oils - Chemical Characterization and Investigation of Some Biological Activities. medicines 3: 1-16.

15) Novak J, Franz C (2003) Composition of the essential oils and extracts of two populations of Cannabis sativa L. Ssp. Spontanea from Austria. Journal of Essential Oil Research 15: 158-160.

16) Klein RFX (2015) Analysis of Marijuana by Liquid Chromatographic Techniques A Literature Survey, 1990 -2015. Microgram Journal 12: 1-4.

17) dos Santos NA, Souza LM, Domingos E, França HS, et al. (2016) Evaluating the selectivity of colorimetric test (Fast Blue $\mathrm{BB}$ salt) for the cannabinoids identification in marijuana street samples by UV-Vis, TLC, ESI(+)FT-ICR MS and ESI(+)MS/MS. Forensic Chemistry 1: 13-21.

18) Hazekamp A, Peltenburg A, Verpoorte R, Giroud C, Murnion, B (2005) Chromatographic and spectroscopic data of cannabinoids from Cannabis sativa L. Australian Prescriber 28: 212-215.

19) Eiceman GA, Karpas Z, Hill Jr. HH (2016) Ion Mobility Spectrometry. Boca Raton, Fl: CRC Press, Taylor \& Francis Group.

20) Cumeras R, Figueras E, Davis CE, Baumbach JI, Gràcia I (2015) Review on Ion Mobility Spectrometry. Part 1: current instrumentation. Analyst 140: 1376-1390.

21) Cumeras R, Figueras E, Davis CE, Baumbach JI, Gràcia I (2015). Review on Ion Mobility Spectrometry. Part 2: hyphenated methods and effects of experimental parameters. Analyst 140: 1391-1410.

22) Ewing RGG, Atkinson DAA, Eiceman GA., Ewing GJJ (2001) A critical review of ion mobility spectrometry for the detection of explosives and explosive related compounds. Talanta 54: 515-529.

23) Mäkinen MA, Anttalainen OA, Sillanpää MET (2010) Ion mobility spectrometry and its applications in the detection of chemical warfare agents. Analytical Chemistry 82: 9594-9600. 24) Vautz W, Zimmermann D, Hartmann M, Baumbach JI, Nolte J, Jung J (2006) Ion mobility spectrometry for food quality and safety. Food Additives \& Contaminants 23: 1064-1073.

25) Baumbach JI (2006) Process analysis using ion mobility spectrometry. Anal. Bioanal. Chem. 384: 1059-1070.

26) Pagonas N, Vautz W, Seifert L, Slodzinski R, Jankowski J, Zidek W, Westhoff TH (2012) Volatile Organic Compounds in Uremia. PLoS One 7: e46258.

27) Perl T, Jünger M, Vautz W, Nolte J, Kuhns M, Borg-von Zepelin M, Quintel M (2011) Detection of characteristic metabolites of Aspergillus fumigatus and Candida species using ion mobility spectrometry - metabolic profiling by volatile organic compounds. Mycoses 54: e828-e837. 
28) Vautz W, Hariharan C, Weigend M (2018) Smell the change: On the potential of gas-chromatographic ion mobility spectrometry in ecosystem monitoring. Ecology and Evolution 8: 4370-4377.

29) Verkouteren JR, Staymates JL (2011) Reliability of ion mobility spectrometry for qualitative analysis of complex, multicomponent illicit drug samples. Forensic Science International 206:190-196.

30) Keller T, Schneider A, Tutsch-Bauer E, Jaspers J, Aderjan R, Skopp G (1992) Ion mobility spectrometry for the detection of drugs in cases of forensic and criminalistic relevance. International Journal for Ion Mobility Spectrometry 2: 22-34.

31) Happyana N, Agnolet S, Muntendam R, Van Dam A, et al. (2013) Analysis of cannabinoids in laser-microdissected trichomes of medicinal Cannabis sativa using LCMS and cryogenic NMR. Phytochemistry 87: 51-59

32) Vautz W, Baumbach JI, Westhoff $M$, Züchner K, Carstens ETH, Perl T (2010) Breath sampling control for medical application. International Journal for Ion Mobility Spectrometry 13: $41-46$

33) Jones AB, ElSohly HN, ElSohly MA (1984) Analysis of the major metabolite of delta 9-tetrahydrocannabinol in the urine. V. Cross-reactivity of selected compounds in a radioimmunoassay. Journal of Analytical Toxicology 8: 252-254.

34) Kintz P, Bernhard W, Villain M, Gasser M, Aebi B, Cirimele V (2005) Detection of cannabis use in drivers with the drug wipe device and by GC-MS after Intercept device collection. Journal of Analytical Toxicology 29:724-727.

35) Pehrsson A, Blencowe T, Vimpari K, Langel K, Engblom C, Lillsunde P (2011) An evaluation of on-site oral fluid drug screening devices drugwipe ${ }^{\bullet} 5$ and rapid STAT ${ }^{\circledast}$ using oral fluid for confirmation analysis. Journal of Analytical Toxicology 35: $211-218$.
Submit your manuscript to a JScholar journal and benefit from:

ฯ Convenient online submission

ๆ Rigorous peer review

9 Immediate publication on acceptance

q Open access: articles freely available online

ฯ High visibility within the field

- Better discount for your subsequent articles

Submit your manuscript at http://www.jscholaronline.org/submit-manuscript.php 